\title{
Nearly Invariant Subspaces with Applications to Truncated Toeplitz Operators
}

\author{
Ryan O'Loughlin ${ }^{1}$
}

Received: 12 May 2020 / Accepted: 13 October 2020 / Published online: 28 October 2020

(c) The Author(s) 2020

\begin{abstract}
In this paper we first study the structure of the scalar and vector-valued nearly invariant subspaces with a finite defect. We then subsequently produce some fruitful applications of our new results. We produce a decomposition theorem for the vector-valued nearly invariant subspaces with a finite defect. More specifically, we show every vectorvalued nearly invariant subspace with a finite defect can be written as the isometric image of a backwards shift invariant subspace. We also show that there is a link between the vector-valued nearly invariant subspaces and the scalar-valued nearly invariant subspaces with a finite defect. This is a powerful result which allows us to gain insight in to the structure of scalar subspaces of the Hardy space using vector-valued Hardy space techniques. These results have far reaching applications, in particular they allow us to develop an all encompassing approach to the study of the kernels of: the Toeplitz operator, the truncated Toeplitz operator, the truncated Toeplitz operator on the multiband space and the dual truncated Toeplitz operator.
\end{abstract}

Keywords Hardy space - Toeplitz operator - Backwards shift operator · Matrix-valued functions

Mathematics Subject Classification $30 \mathrm{H} 10 \cdot 47 \mathrm{~B} 35 \cdot$ 46E15 · 47A56

\section{Introduction}

The purpose of this paper is to study vector and scalar-valued nearly $S^{*}$-invariant subspaces of the Hardy space defined on the unit disc. We first produce some results on the structure of nearly $S^{*}$-invariant subspaces with a finite defect, in particular we

\section{Communicated by Isabelle Chalendar.}

\footnotetext{
$凶 \quad$ Ryan O'Loughlin mm12rol@leeds.ac.uk

1 School of Mathematics, University of Leeds, Leeds LS2 9JT, UK
} 
produce a powerful tool which allows us to relate the vector-valued nearly $S^{*}$-invariant subspaces to scalar-valued nearly $S^{*}$-invariant subspaces with a finite defect. These results then allow us to adopt a previously unknown universal approach to the study of the kernel of: the Toeplitz operator, the truncated Toeplitz operator, the dual truncated Toeplitz operator and the truncated Toeplitz operator on the multiband space (all to be defined later).

We denote $\mathbb{T}$ to be the unit circle and $\mathbb{D}$ to be the open unit disc. The vector-valued Hardy space is denoted $H^{2}\left(\mathbb{D}, \mathbb{C}^{n}\right)$ and is the Hilbert space defined to be a column vector of length $n$ with each coordinate taking values in $H^{2}$; background theory on the classical Hardy space $H^{2}$ can be found in $[12,16]$. The backwards shift on the space $H^{2}\left(\mathbb{D}, \mathbb{C}^{n}\right)$ is defined by

$$
S^{*}\left(\begin{array}{c}
f_{1} \\
\vdots \\
f_{n}
\end{array}\right)(z)=\frac{\left(\begin{array}{c}
f_{1}(z) \\
\vdots \\
f_{n}(z)
\end{array}\right)-\left(\begin{array}{c}
f_{1}(0) \\
\vdots \\
f_{n}(0)
\end{array}\right)}{z} .
$$

If we denote $\overline{H_{0}^{2}}=\left\{\bar{f}: f \in H^{2}, f(0)=0\right\}$, then it is readily checked that $\overline{H_{0}^{2}}$ is the orthogonal complement of $H^{2}$ in $L^{2}(\mathbb{T})$. Then in the scalar case (i.e. when $n=1$ ) using Beurling's Theorem one can then deduce that all non-trivial $S^{*}$-invariant subspaces are of the form $K_{\theta}=\theta \overline{H_{0}^{2}} \cap H^{2}$ for some inner function $\theta$. We call $K_{\theta}$ a model space and further information on model spaces can be found in [7]. One can further check that for distinct $\lambda_{i} \in \mathbb{D}$ if $\theta=\prod_{i} \frac{z-\lambda_{i}}{1-\overline{\lambda_{i} z}}$, then $K_{\theta}$ is the span of Cauchy kernels $k_{\lambda_{i}}(z)=\sum_{n=0}^{\infty}\left(\overline{\lambda_{i}} z\right)^{n}$. The Cauchy kernel $k_{\lambda_{i}}$ is the eigenvector of the backwards shift with eigenvalue $\overline{\lambda_{i}}$.

Definition 1.1 A closed subspace $M \subseteq H^{2}\left(\mathbb{D}, \mathbb{C}^{n}\right)$ is said to be nearly $S^{*}$-invariant with defect $m$ if and only if there exists a $m$-dimensional subspace $D$ (which may be taken to be orthogonal to $M$ ) such that if $f \in M$ and $f(0)$ is the zero vector then $S^{*} f \in M \oplus D$.

If $M$ is nearly $S^{*}$-invariant with defect 0 then it is said to be nearly $S^{*}$-invariant.

Using orthogonal decomposition we can write $L^{2}=\overline{H_{0}^{2}} \oplus K_{\theta} \oplus \theta H^{2}$. We define $P_{\theta}: L^{2} \rightarrow K_{\theta}$ to be the orthogonal projection.

Definition 1.2 The truncated Toeplitz operator $A_{g}^{\theta}: K_{\theta} \rightarrow K_{\theta}$ having symbol $g \in L^{2}$ is the densely defined operator

$$
A_{g}^{\theta}(f)=P_{\theta}(g f)
$$

having domain

$$
\left\{f \in K_{\theta}: g f \in L^{2}\right\}
$$


The concept of (scalar) nearly backwards shift invariant subspaces was first introduced by Hitt in [15] as a generalisation to Hayashi's results concerning Toeplitz kernels in [14]. These spaces were then studied further by Sarason [19]. The study of nearly backwards shift invariant subspaces was then generalised to the vectorial case in [5], and generalised to include a finite defect in [6]. Kernels of Toeplitz operators are the prototypical example of nearly $S^{*}$ - invariant subspaces.

Truncated Toeplitz operators were introduced in [20], and over the past decade there have been many further publications studying their properties. The applications of truncated Toeplitz operators are vast, ranging from purely mathematical to more applied problems. From a purely mathematical perspective one can use the Sz.-NagyFoiaş model theory for Hilbert space contractions (see [16]) to show that every Hilbert space contraction $T$ having defect indices $(1,1)$ and such that $\lim _{n \rightarrow \infty}\left(T^{*}\right)^{n}$ (SOT) is unitarily equivalent to $A_{z}^{\theta}$, for some inner function $\theta$. This can be generalised to produce similar results for arbitrary defect indices. Another notable application of truncated Toeplitz operators within pure mathematics comes from the Carathéodory and Pick problems [1], where truncated Toeplitz operators with an analytic symbol appear naturally. From a more applied perspective truncated Toeplitz operators have links to control theory and electrical engineering. More specifically when one is considering an extremal problem posed over $H^{\infty}$, the solution of such a problem can be solved by computing the norm of a Hankel operator, and the norm of the Hankel operator can in turn be shown to equal the norm of an analytic truncated Toeplitz operator. This is shown explicitly as equation 2.9 in [18].

Although truncated Toeplitz operators share many properties with the classical Toeplitz operator it is easily checked that the kernel of a truncated Toeplitz operator is not nearly $S^{*}$-invariant. This motivates our study for Sect. 2 where we show under certain conditions the kernel of a truncated Toeplitz operator is in fact nearly $S^{*}$ invariant with defect 1 . In many cases the study of Toeplitz operators becomes greatly simplified when the operator has an invertible symbol; in Sect. 2 we also show that the symbol of a truncated Toeplitz operator may be chosen to be invertible in $L^{\infty}$.

In Sect. 3 we prove a powerful result that shows for any $i \in\{1 \ldots n\}$ the first $i$ coordinates of a vector-valued nearly $S^{*}$-invariant subspace is a nearly $S^{*}$-invariant subspace with a finite defect. We then generalise Theorem 3.2 in [5] and Corollary 4.5 in [6] to find a Hitt-style decomposition for the vector-valued nearly $S^{*}$-invariant subspaces with a finite defect.

In Sect. 4 we show that in all cases the kernel of a truncated Toeplitz operator is a nearly $S^{*}$-invariant subspace with defect 1 ; this then allows us to decompose the kernel in to an isometric image of a model space. The approach of decomposing a kernel in to an isometric image of a model space much resembles the works of Hayashi [14] and Hitt [15] for the classical Toeplitz operator. We also make the observation that we can decompose the kernel of a truncated Toeplitz operator in to a nearly $S^{*}$-invariant subspace multiplied by a power of $z$ (where $z \in \mathbb{D}$ is the independent variable). Then using the results of [15], this observation also gives us a second method to decompose the kernel in to a isometric image of a model space. Furthermore we show that in general our two choices of decomposition of the kernel of a truncated Toeplitz operator yield different results. 
In Sect. 5 we study the kernel of dual truncated Toeplitz operator. Dual truncated Toeplitz operators have been studied in both $[9,11]$ as well as many other sources. The kernel of a dual truncated Toeplitz operator has been studied in [8]. Although the domain of the dual truncated Toeplitz operator is not a subspace of $H^{2}$ we still can use similar recursive techniques used in previous sections to decompose the the kernel in to a fixed function multiplied by a $S^{*}$-invariant subspace.

In Sect. 6 we study the truncated Toeplitz operator on the multiband space. We show every truncated Toeplitz operator on a multiband space is unitarily equivalent to an operator which has kernel nearly $S^{*}$-invariant with defect 2 . This allows us to apply our previously developed theory to give a decomposition for the kernel of the truncated Toeplitz operator on a multiband space in terms of $S^{*}$-invariant subspaces.

\subsection{Notations and Convention}

- From Sect. 3 onward we assume the symbol of any Toeplitz operator (denoted $g$ ) is bounded and hence the Toeplitz operator is bounded.

- Throughout we let $\theta$ be an arbitrary inner function.

- We use the notation $f^{i} / f^{o}$ to denote the inner/outer factor of $f$.

- $G C D$ stands for greatest common divisor, and the greatest common divisor of two inner functions is always taken to be an inner function.

- All limits are taken in the $H^{2}\left(\mathbb{D}, \mathbb{C}^{n}\right)$ sense unless otherwise stated.

- All subspaces of $H^{2}\left(\mathbb{D}, \mathbb{C}^{n}\right)$ are assumed closed unless otherwise stated.

\section{Preliminary Results}

Theorem 2.1 For any $g \in L^{2}$ we write $g=g^{-}+g^{+}$where $g^{-} \in \overline{H_{0}^{2}}$ and $g^{+} \in H^{2}$. If $\overline{g^{-}}$is not cyclic for the backwards shift then there exists a $\tilde{g} \in L^{2}$ such that $A_{g}^{\theta}=A_{\tilde{g}}^{\theta}$ and $\tilde{g}^{-1} \in H^{\infty}$.

Proof Theorem 3.1 of [20] shows that $A_{g_{1}}^{\theta}=A_{g_{2}}^{\theta}$ if and only if $g_{1}-g_{2} \in \overline{\theta H^{2}}+\theta H^{2}$, so we may initially assume without loss of generality that $g \in \overline{K_{\theta}} \oplus K_{\theta}$. Using Lemma 2.1 in [17] we can construct an outer function $u$ such that $|u|=2|g|+1$, furthermore $u \in L^{2}$ so $u \in H^{2}$. Then it follows that for any inner function $\alpha$

$$
g-\overline{\alpha u}
$$

has the property that

$$
|g-\overline{\alpha u}| \geq|u|-|g|>|g|+1>0
$$

almost everywhere on $\mathbb{T}$, and so $(g-\overline{\alpha u})^{-1} \in L^{\infty}$. Our construction of $u$ shows $\left|\frac{1}{u}\right| \leq 1$ and as the reciprocal of an outer function in is outer, we have $\frac{1}{u}$ is outer and in $L^{\infty}$, so $\frac{1}{u} \in H^{\infty}$. Furthermore by Corollary 4.9 in [17] we can say $\frac{1}{u} \in H^{2}$ is non-cyclic for $S^{*}$ and hence must lie in a model space $K_{\Phi}$. Define $\tilde{g}:=(g-\overline{\Phi \theta u})$, 
then as previously stated $\tilde{g}^{-1} \in L^{\infty}$. We now show $\tilde{g}^{-1}=\sum_{k=0}^{\infty}(-1) g^{k}\left(\Phi \theta \frac{1}{\bar{u}}\right)^{k+1}$ where the limit is taken in the sense of uniform convergence. We write $\tilde{g}_{N}^{-1}$ to be $\sum_{k=0}^{N}(-1) g^{k}\left(\Phi \theta \frac{1}{\bar{u}}\right)^{k+1}$ then we have $\left\|\tilde{g}_{N}^{-1}-\tilde{g}^{-1}\right\|_{\infty}$ is equal to

$$
\left\|\tilde{g}^{-1} \tilde{g}\left(\tilde{g}_{N}^{-1}-\tilde{g}^{-1}\right)\right\|_{\infty} \leq\left\|\tilde{g}^{-1}\right\|_{\infty}\left\|\tilde{g}^{-1} \tilde{g}_{N}^{-1}-1\right\|_{\infty} \leq\left\|\tilde{g}^{-1}\right\|_{\infty}\left\|g^{N}\left(\Phi \theta \frac{1}{\bar{u}}\right)^{N}\right\|_{\infty} .
$$

By our construction of $u$ this is less than $\left\|\tilde{g}^{-1}\right\|_{\infty}\left(\frac{1}{2}\right)^{N}$, which clearly converges to 0 . Now our choice of $\Phi$ ensures that $\Phi \frac{1}{\bar{u}} \in H^{\infty}$, we also have $\theta g \in H^{2}$. This means $(-1) g^{k}\left(\Phi \theta \frac{1}{\bar{u}}\right)^{k+1} \in H^{2}$ and is bounded by 1 so must actually lie in $H^{\infty}$, so $\tilde{g}^{-1}$ (being the uniform limit of a sequence in $H^{\infty}$ ) must also be in $H^{\infty}$.

Examining the first part of the above proof we can also deduce the following proposition.

Proposition 2.2 For any $g \in L^{2}$ there exists a $\tilde{g} \in L^{2}$ such that $A_{g}^{\theta}=A_{\tilde{g}}^{\theta}$ and $\tilde{g}^{-1} \in L^{\infty}$.

Proof In (1) if we set $\alpha$ to equal $\theta$, keep our construction of $u$ the same and define $\tilde{g}=g-\overline{\alpha u}$ then $A_{g}^{\theta}=A_{\tilde{g}}^{\theta}$. Furthermore the computation immediately after (1) shows $\tilde{g}^{-1} \in L^{\infty}$.

This has an interesting relation to Sarason's question posed in [20]; which is whether every bounded truncated Toeplitz operator has a bounded symbol. This was first shown to have a negative answer as Theorem 5.3 in [3], and further results in [2] characterise the inner functions $\theta$ which have the property that every bounded truncated Toeplitz operator on $K_{\theta}$ has a bounded symbol.

These results suggest that under certain circumstances $\operatorname{ker} A_{g}^{\theta}$ may be a nearly invariant subspace with a finite defect. This is because $f \in \operatorname{ker} A_{g}^{\theta}$ if and only if $f \in K_{\theta}$ and

$$
g f \in \overline{H_{0}^{2}} \oplus \theta H^{2}
$$

so if $f(0)=0$ and $f \in \operatorname{ker} A_{g}^{\theta}$ then we must have

$$
\frac{g f}{z} \in \overline{H_{0}^{2}}+\operatorname{span}\left\{S^{*}(\theta)\right\}+\theta H^{2}
$$

This may lead us to believe that $\operatorname{ker} A_{g}^{\theta}$ is a nearly $S^{*}$-invariant subspace with a defect given by $g^{-1} \operatorname{span}\left\{S^{*}(\theta)\right\}$, but the issue here is $g^{-1} S^{*}(\theta)$ need not necessarily lie in $K_{\theta}$ or even $H^{2}$. Theorem 2.1 shows us that under some weak restrictions we can choose our non-unique symbol $g$ so that $g^{-1} S^{*}(\theta) \in H^{2}$, but to fully understand ker $A_{g}^{\theta}$ as a nearly invariant subspace with a defect we must study vector-valued nearly invariant subspaces with a defect. 


\section{Vector-Valued Nearly Invariant Subspaces with a Defect}

Let $M \subseteq H^{2}\left(\mathbb{D}, \mathbb{C}^{n}\right)$ be a nearly invariant subspace for the backwards shift with a finite defect space $D$ and let $\operatorname{dim} D=m$. If not all functions in $M$ vanish at 0 then we define $W:=M \ominus\left(M \cap z H^{2}\left(\mathbb{D}, \mathbb{C}^{n}\right)\right)$ and Corollary 4.3 in [5] shows that $r:=\operatorname{dim} W \leq n$, in this case we let $W_{1} \ldots W_{r}$ be an orthonormal basis of $W$. For $i=1 \ldots n$ we let $P_{i}: H^{2}\left(\mathbb{D}, \mathbb{C}^{n}\right) \rightarrow H^{2}\left(\mathbb{D}, \mathbb{C}^{i}\right)$ be the projection on to the first $i$ coordinates.

Theorem 3.1 For any $i \in\{1 \ldots n\}, M_{i}:=P_{i}(M)$ is a (not necessarily closed) nearly invariant subspace with a defect space $\left(\frac{\operatorname{span}\left\{P_{i}\left(W_{1}\right), \ldots P_{i}\left(W_{r}\right)\right\}}{z} \cap H^{2}\left(\mathbb{D}, \mathbb{C}^{i}\right)\right)+P_{i}(D)$.

Proof We first consider the case when not all functions in $M$ vanish at 0 . Let $f_{i} \in M_{i}$, then $f_{i}$ is the first $i$ entries of some $F \in M$. We write $F$ as

$$
F=a_{1} W_{1}+\ldots a_{r} W_{r}+F_{1}
$$

where $a_{1} \ldots a_{r} \in \mathbb{C}$ and $F_{1} \in M \cap z H^{2}\left(\mathbb{D}, \mathbb{C}^{n}\right)$. So if $f_{i}(0)$ is the zero vector, we then have $f_{i}(0)$ is zero and $F_{1}(0)$ is zero, which forces $P_{i}\left(a_{1} W_{1}+\ldots a_{r} W_{r}\right)$ to be zero. So

$$
\frac{f_{i}}{z}-\frac{P_{i}\left(a_{1} W_{1}+\ldots a_{r} W_{r}\right)}{z}=P_{i}\left(\frac{F_{1}}{z}\right) \in M_{i}+P_{i}(D),
$$

which means

$$
\frac{f_{i}}{z} \in M_{i}+\left(\frac{\operatorname{span}\left\{P_{i}\left(W_{1}\right), \ldots P_{i}\left(W_{r}\right)\right\}}{z} \cap H^{2}\right)+P_{i}(D) .
$$

In the case when all functions in $M$ vanish at 0 then $W=\{0\}$ and we would just have $\frac{F}{z} \in M+D$, so $\frac{f_{i}}{z} \in M_{i}+P_{i}(D)$.

Remark If $W=\{0\}$ we can interpret $\left(\frac{\operatorname{span}\left\{P_{i}\left(W_{1}\right), \ldots P_{i}\left(W_{r}\right)\right\}}{z} \cap H^{2}\left(\mathbb{D}, \mathbb{C}^{2}\right)\right)$ to be the zero vector.

Corollary 3.2 With the same assumptions as in Theorem 3.1, if $m=0$ i.e. if $M$ is a nearly $S^{*}$-invariant subspace, then $M_{i}$ is a (not necessarily closed) nearly $S^{*}$-invariant subspace with a defect space $\left(\frac{\operatorname{span}\left\{P_{i}\left(W_{1}\right), \ldots P_{i}\left(W_{r}\right)\right\}}{z} \cap H^{2}\left(\mathbb{D}, \mathbb{C}^{i}\right)\right)$.

To further build on this result we will now give a Hitt style decomposition for a vector-valued nearly invariant subspace with a finite defect. This style of decomposition was first introduced by Hitt in [15] when he decomposed the nearly $S^{*}$-invariant subspaces. This was then generalised to the vectorial case as Corollary 4.5 in [5]. This style of proof was then adapted to produce a similar result for the (scalar) nearly invariant subspace with a defect, which is Theorem 3.2 in [6].

For a Hilbert space $\mathcal{H}$ and $x, y \in \mathcal{H}$ we define $x \otimes y(f)=\langle f, y\rangle x$. We say an operator $T$ on $\mathcal{H}$ belongs to the class $C_{.0}$ if for all $x \in \mathcal{H}, \lim _{n \rightarrow \infty}\left\|\left(T^{*}\right)^{n} x\right\|=0$. 
Consider a subspace $M$ which is nearly $S^{*}$-invariant with defect 1 , so that $D=$ $\operatorname{span}\left\{e_{1}\right\}$, say, where $\left\|e_{1}\right\|=1$.

Suppose first that not all functions in $M$ vanish at 0 , then $1 \leq r=\operatorname{dim} W \leq n$. Let $F_{0}$ be the matrix with columns $W_{1} \ldots W_{r}$, and let $P_{W}$ be the orthogonal projection on to $W$. For each $F \in M$ we may write

$$
F=P_{W}(F)+F_{1}=F_{0}\left(\begin{array}{c}
a_{0}^{1} \\
\vdots \\
a_{0}^{r}
\end{array}\right)+F_{1}
$$

Now as $F_{1}(0)=0$ we have $S^{*}\left(F_{1}\right)=G_{1}+\beta_{1} e_{1}$, where $G_{1} \in M$ and $\beta_{1} \in \mathbb{C}$. Thus

$$
F(z)=F_{0}(z) A_{0}+z G_{1}(z)+z \beta e_{1}(z)
$$

where $A_{0}=\left(\begin{array}{c}a_{0}^{1} \\ \vdots \\ a_{0}^{r}\end{array}\right)$. Moreover since the family $\left\{W_{i}\right\}_{i=1 \ldots r}$ forms an orthonormal basis of $W$, we obtain the following identity of norms:

$$
\|F\|^{2}=\left\|F_{0} A_{0}\right\|^{2}+\left\|F_{1}\right\|^{2}=\left\|A_{0}\right\|^{2}+\left\|G_{1}\right\|^{2}+\left|\beta_{1}\right|^{2}
$$

We may now repeat this process on $G_{1}$ to obtain $G_{1}=P_{W}\left(G_{1}\right)+F_{2}$, and $S^{*}\left(F_{2}\right)=$ $G_{2}+\beta_{2} e_{1}$, so $G_{1}=F_{0} A_{1}+z G_{2}+z \beta_{2} e_{1}$. We iterate this process to obtain

$$
F(z)=F_{0}(z)\left(A_{0}+A_{1} z+\ldots A_{n-1} z^{n-1}\right)+z G_{n}(z)+\left(\beta_{1} z+\ldots+\beta_{n} z^{n}\right) e_{1}(z)(2)
$$

where

$$
\|F\|^{2}=\sum_{k=0}^{n-1}\left\|A_{k}\right\|^{2}+\left\|G_{n}\right\|^{2}+\sum_{k=1}^{n}\left|\beta_{k}\right|^{2} .
$$

We now argue $\left\|G_{n}\right\| \rightarrow 0$ as $n \rightarrow \infty$. We can write $G_{n}=P_{1} S^{*} P_{2}\left(G_{n-1}\right)$, where $P_{1}$ is the projection with kernel $\left\langle e_{1}\right\rangle$ and $P_{2}$ is the projection with kernel $\operatorname{span}\left\{W_{1} \ldots W_{r}\right\}$. For all $n \geq 1$ we may write $G_{n+1}=P_{1} R^{n-1}\left(S^{*} P_{2}\left(G_{1}\right)\right)$, where $R=S^{*} P_{2} P_{1}$ and so

$$
\left\|G_{n+1}\right\| \leq\left\|P_{1}\right\|\left\|R^{n-1}\left(S^{*} P_{2}\left(G_{1}\right)\right)\right\| .
$$

As $e_{1}$ is orthogonal to $W$ we have

$$
P_{2} P_{1}=P_{1} P_{2}=I d-e_{1} \otimes e_{1}-\sum_{j=1}^{r} W_{j} \otimes W_{j},
$$


and so the adjoint of $R$ is

$$
P_{1} P_{2} S=S-e_{1} \otimes S^{*}\left(e_{1}\right)-\sum_{j=1}^{r} W_{j} \otimes S^{*}\left(W_{j}\right)
$$

We now apply the second assertion of Proposition 2.1 from [5] to show the adjoint of $R$ is of class $C_{.0}$, and so $R^{n-1}$ applied to $S^{*} P_{2}\left(G_{1}\right)$ converges to 0 ; now from (3) we see $\left\|G_{n+1}\right\| \rightarrow 0$. As a consequence taking limits in (2) we may write

$$
F(z)=\lim _{n \rightarrow \infty}\left(F_{0}(z)\left(A_{0}+A_{1} z+\ldots A_{n-1} z^{n-1}\right)+\left(\beta_{1} z+\ldots+\beta_{n} z^{n}\right) e_{1}(z)\right) .
$$

We denote $a_{n}(z)=F_{0}(z)\left(A_{0}+A_{1} z+\ldots A_{n-1} z^{n-1}\right)$, and $a_{0}(z)=F_{0}\left(\sum_{k=0}^{\infty} A_{k} z^{k}\right)$, where $\left(\sum_{k=0}^{\infty} A_{k} z^{k}\right)$ is taken in the $H^{2}\left(\mathbb{D}, \mathbb{C}^{n}\right)$ sense (this is defined by the equality of norms given immediately after (2)). Then in the $H^{1}\left(\mathbb{D}, \mathbb{C}^{n}\right)$ norm we must have

$$
\left\|a_{n}(z)-a_{0}(z)\right\|=\left\|F_{0} \sum_{k=n}^{\infty} A_{k} z^{k}\right\| \leq\left\|W_{1} \sum_{k=n}^{\infty} a_{k}^{1} z^{k}\right\|+\ldots+\left\|W_{r} \sum_{k=n}^{\infty} a_{k}^{r} z^{k}\right\|
$$

For each $i \in\{1 \ldots n\}$ we define $C_{i}$ to equal the maximum $H^{2}$ norm of each coordinate of $W_{i}$ multiplied by $n$, then we apply Hölder's inequality on each coordinate to obtain

$$
\left\|W_{i} \sum_{k=n}^{\infty} a_{k}^{i} z^{k}\right\|_{H^{1}\left(\mathbb{D}, \mathbb{C}^{n}\right)} \leq C_{i}\left\|\sum_{k=n}^{\infty} a_{k}^{i} z^{k}\right\|_{H^{2}\left(\mathbb{D}, \mathbb{C}^{n}\right)} \rightarrow 0
$$

Thus in the $H^{1}\left(\mathbb{D}, \mathbb{C}^{n}\right)$ norm we have $a_{n} \rightarrow a_{0}$, a similar computation shows $\left(\beta_{1} z+\ldots+\beta_{n} z^{n}\right) e_{1}(z)$ converges to $\left(\sum_{k=1}^{\infty} \beta_{k} z^{k}\right) e_{1}$ in the $H^{1}\left(\mathbb{D}, \mathbb{C}^{n}\right)$ norm, so the $H^{1}\left(\mathbb{D}, \mathbb{C}^{n}\right)$ limit of

$$
F(z)=F_{0}(z)\left(A_{0}+A_{1} z+\ldots A_{n-1} z^{n-1}\right)+\left(\beta_{1} z+\ldots+\beta_{n} z^{n}\right) e_{1}(z)
$$

must be equal to

$$
F(z)=F_{0}\left(\sum_{k=0}^{\infty} A_{k} z^{k}\right)+\left(\sum_{k=1}^{\infty} \beta_{k} z^{k}\right) e_{1}
$$

and furthermore by taking limits in the equality of norms immediately after (2) we know

$$
\|F\|^{2}=\sum_{k=0}^{\infty}\left\|A_{k}\right\|^{2}+\sum_{k=1}^{\infty}\left|\beta_{k}\right|^{2}
$$


We may alternatively express this as saying $F \in M$ if and only if

$$
F(z)=F_{0} k_{0}+z k_{1} e_{1}
$$

where $\left(k_{0}, k_{1}\right)$ lies in a subspace $K \subseteq H^{2}\left(\mathbb{D}, \mathbb{C}^{r}\right) \times H^{2}$ which is identified with $H^{2}\left(\mathbb{D}, \mathbb{C}^{r+1}\right)$.

By virtue of (4) we can see that $K$ is the image of a isometric mapping, and hence closed. We now argue $K$ is invariant under the backwards shift (on $H^{2}\left(\mathbb{D}, \mathbb{C}^{r+1}\right)$ ). Since in the algorithm we have $k_{0}(0)=A_{0}$ and $k_{1}(0)=\beta_{1}$ we can write $F$ as

$$
F=F_{0} A_{0}+z F_{0} S^{*}\left(k_{0}\right)+\beta_{1} z e_{1}+z^{2} S^{*}\left(k_{1}\right) e_{1},
$$

consequently

$$
F_{0} S^{*}\left(k_{0}\right)+z S^{*}\left(k_{1}\right) e_{1}=\frac{F-F_{0} A_{0}-\beta_{1} z e_{1}}{z}=G_{1} \in M .
$$

Conversely if

$$
M=\left\{F_{0} k_{0}+z k_{1} e_{1}:\left(k_{0}, k_{1}\right) \in K\right\},
$$

is a closed subspace of $H^{2}\left(\mathbb{D}, \mathbb{C}^{n}\right)$, where $K$ is a $S^{*}$-invariant subspace of $H^{2}\left(\mathbb{D}, \mathbb{C}^{r+1}\right)$, then $M$ is nearly $S^{*}$-invariant with defect 1 . To show this we first need a lemma.

Lemma $3.3 W_{1}(0), \ldots W_{r}(0)$ are linearly independent in $\mathbb{C}^{n}$.

Proof If $W_{k}(0)=\sum_{i \neq k} \lambda_{i} W_{i}(0)$ this would mean $W_{k}-\sum_{i \neq k} \lambda_{i} W_{i}$ vanishes at 0 and therefore lies in $z H^{2}\left(\mathbb{D}, \mathbb{C}^{n}\right)$.

If $F \in M$ and $F(0)=0$ then we must have $F_{0}(0) k_{0}(0)$ is equal to the zero vector. We now add $n-r$ vectors $X_{1} \ldots X_{n-r}$ which are linearly independent from $W_{1}(0), \ldots W_{r}(0)$ as extra columns to the matrix $F_{0}(0)$ to obtain a matrix

$$
F_{0}^{\prime}(0)=\left[W_{1}, \ldots W_{r}, X_{1}, \ldots, X_{n-r}\right]
$$

We now add $n-r$ extra 0 's to the end of the column vector $k_{0}(0)$ and label this $k_{0}^{\prime}(0)$. As $F_{0}(0) k_{0}(0)$ is equal to the zero vector, then $F_{0}^{\prime}(0) k_{0}^{\prime}(0)$ must also be equal to the zero vector. We can now invert $F_{0}^{\prime}(0)$ to obtain $k_{0}^{\prime}(0)$ is equal to the zero vector and hence $k_{0}(0)$ must be zero. This allows us to write

$$
S^{*}(F)=F_{0} \frac{k_{0}}{z}+k_{1} e_{1}=F_{0} \frac{k_{0}}{z}+z S^{*} k_{1} e_{1}+k_{1}(0) e_{1},
$$

and as $K$ is $S^{*}$-invariant this is clearly an element of $M \oplus \operatorname{span}\left\{e_{1}\right\}$. 
If all functions in $M$ vanish at 0 then there is no non-trivial reproducing kernel at 0 , but we may now write

$$
F(z)=z\left(G_{1}(z)+\beta_{1} e_{1}(z)\right)
$$

with $G_{1} \in M$ and $\beta_{1} \in \mathbb{C}$, and furthermore

$$
\|F\|^{2}=\left\|G_{1}\right\|^{2}+\left|\beta_{1}\right|^{2} .
$$

We can then iterate on $G_{1}$ as we have previously done to obtain

$$
F(z)=\beta_{1} z e_{1}+\beta_{2} z^{2} e_{1}+\ldots
$$

For a general finite defect $m$ the analogous calculations produce the following result.

Theorem 3.4 Let $M$ be a closed subspace that is nearly $S^{*}$-invariant with a finite defect $m$. Then:

1. In the case where there are functions in $M$ that do not vanish at 0 ,

$$
M=\left\{F: F(z)=F_{0}(z) k_{0}(z)+z \sum_{j=1}^{m} k_{j}(z) e_{j}(z):\left(k_{0}, \ldots, k_{m}\right) \in K\right\}
$$

where $F_{0}$ is the matrix with each column being an orthonormal element of $W$, $\left\{e_{1}, \ldots e_{m}\right\}$ is any orthonormal basis for $D, k_{0} \in H^{2}\left(\mathbb{D}, \mathbb{C}^{r}\right)($ where $r=\operatorname{dim} W)$, $k_{1}, \ldots k_{m} \in H^{2}$, and $K \subseteq H^{2}\left(\mathbb{D}, \mathbb{C}^{(r+m)}\right)$ is a closed $S^{*}$-invariant subspace. Furthermore $\|F\|^{2}=\sum_{j=0}^{m}\left\|k_{j}\right\|^{2}$.

2. In the case where all functions in $M$ vanish at 0 ,

$$
M=\left\{F: F(z)=z \sum_{j=1}^{m} k_{j}(z) e_{j}(z):\left(k_{1}, \ldots, k_{m}\right) \in K\right\}
$$

with the same notation as in 1 , except that $K$ is now a closed $S^{*}$-invariant subspace of $H^{2}\left(\mathbb{D}, \mathbb{C}^{m}\right)$, and $\|F\|^{2}=\sum_{j=1}^{m}\left\|k_{j}\right\|^{2}$.

Conversely if a closed subspace $M \subseteq H^{2}\left(\mathbb{D}, \mathbb{C}^{n}\right)$ has a representation as in 1 or 2 , the it is a nearly $S^{*}$-invariant subspace with defect $m$.

\section{Application to Truncated Toeplitz Operators}

Throughout this section our symbol $g$ is bounded and so the truncated Toeplitz operator $A_{g}^{\theta}: K_{\theta} \rightarrow K_{\theta}$ is defined by

$$
A_{g}^{\theta}(f)=P_{\theta}(g f)
$$


where $P_{\theta}$ is the orthogonal projection $L^{2} \rightarrow K_{\theta}$.

It was originally observed in [4] that the kernel of a truncated Toeplitz operator is the first coordinate of the kernel of the matricial Toeplitz operator with symbol

$$
G=\left(\begin{array}{ll}
\bar{\theta} & 0 \\
g & \theta
\end{array}\right)
$$

Scalar-type Toeplitz kernels (first introduced in [10]) are vector-valued Toeplitz kernels which can be expressed as the product of a space of scalar functions by a fixed vector function. A maximal function for $\operatorname{ker} T_{G}$ is an element $f \in \operatorname{ker} T_{G}$ such that if $f \in$ ker $T_{H}$ for any other bounded matricial symbol $H$, then $\operatorname{ker} T_{G} \subseteq \operatorname{ker} T_{H}$. By Corollary 3.9 in [10] $\operatorname{ker} T_{G}$ is of scalar type, it is also easily checked that $\operatorname{ker} T_{G}$ is not shift invariant and so by Theorem 3.7 in [10] we must have that $\operatorname{ker} T_{G}$ has a maximal function. Now by Theorem 3.10 of [17] we can deduce that $W=\operatorname{ker} T_{G} \ominus\left(\operatorname{ker} T_{G} \cap\right.$ $\left.z H^{2}\left(\mathbb{D}, \mathbb{C}^{n}\right)\right)$ has dimension 1 . If we denote $\left(\begin{array}{l}w_{1} \\ w_{2}\end{array}\right)$ to be the normalised element of $W$ then using Corollary 4.5 from [5] we can write

$$
\operatorname{ker} T_{G}=\left(\begin{array}{l}
w_{1} \\
w_{2}
\end{array}\right) K_{z} \Phi
$$

where $\Phi$ is an inner function. We now can write

$$
\operatorname{ker} A_{g}^{\theta}=w_{1} K_{z \Phi}
$$

We can describe $\Phi$ with the following proposition.

Proposition 4.1 When $\operatorname{ker} T_{G}=\left(\begin{array}{l}w_{1} \\ w_{2}\end{array}\right) K_{z} \Phi, \Phi$ is the unique (up to multiplication by a unimodular constant) inner function for which there exists $p_{1}, p_{2} \in H^{2}$ such that

$$
G\left(\begin{array}{l}
w_{1} \\
w_{2}
\end{array}\right) \Phi=\left(\frac{\overline{z p_{1}}}{z p_{2}}\right),
$$

and $G C D\left(p_{1}^{i}, p_{2}^{i}\right)=1$.

Proof We first show that up to multiplication by a unitary constant there can only be one inner function $\Phi$ satisfying

$$
G\left(\begin{array}{l}
w_{1} \\
w_{2}
\end{array}\right) \Phi=\left(\frac{\overline{z p_{1}}}{z p_{2}}\right),
$$

where $G C D\left(p_{1}^{i}, p_{2}^{i}\right)=1$. Suppose there are two inner functions $\Phi_{1}, \Phi_{2}$ such that

$$
G\left(\begin{array}{l}
w_{1} \\
w_{2}
\end{array}\right) \Phi_{1}=\left(\frac{\overline{z p_{1}}}{z p_{2}}\right)
$$


and

$$
G\left(\begin{array}{l}
w_{1} \\
w_{2}
\end{array}\right) \Phi_{2}=\left(\frac{\overline{z q 1}}{z q_{2}}\right)
$$

where both $G C D\left(p_{1}^{i}, p_{2}^{i}\right)=1$ and $G C D\left(q_{1}^{i}, q_{2}^{i}\right)=1$. This would then imply that

$$
\overline{\Phi_{1}}\left(\frac{\overline{z p_{1}}}{z p_{2}}\right)=\overline{\Phi_{2}}\left(\frac{\overline{z q_{1}}}{z q_{2}}\right)
$$

and so $\left(\Phi_{1} p_{1}\right)^{i}=\left(\Phi_{2} q_{1}\right)^{i}$ and $\left(\Phi_{1} p_{2}\right)^{i}=\left(\Phi_{2} q_{2}\right)^{i}$. By assumption we have $G C D\left(p_{1}^{i}, p_{2}^{i}\right)=1$ so $G C D\left(\left(\Phi_{1} p_{2}\right)^{i},\left(\Phi_{1} p_{1}\right)^{i}\right)=\Phi_{1}$, but substituting $\left(\Phi_{1} p_{1}\right)^{i}$ for $\left(\Phi_{2} q_{1}\right)^{i}$ we obtain

$$
G C D\left(\left(\Phi_{1} p_{2}\right)^{i},\left(\Phi_{2} q_{1}\right)^{i}\right)=\Phi_{1},
$$

and so $\Phi_{1}$ divides $\Phi_{2}$. A similar computation shows $\Phi_{2}$ divides $\Phi_{1}$, and so we must have $\Phi_{1}$ is a unitary constant multiple of $\Phi_{2}$. We now show that $\Phi$ is such that

$$
G\left(\begin{array}{l}
w_{1} \\
w_{2}
\end{array}\right) \Phi=\left(\frac{\overline{z p_{1}}}{z p_{2}}\right)
$$

with $G C D\left(p_{1}^{i}, p_{2}^{i}\right)=1$. If it is the case that $\alpha=G C D\left(p_{1}^{i}, p_{2}^{i}\right) \neq 1$ then it would follow that $\left(\begin{array}{l}w_{1} \\ w_{2}\end{array}\right) \Phi \alpha \in \operatorname{ker} T_{G}$, which would be a contradiction as $\Phi \alpha \notin K_{z} \Phi$.

It is easily checked that $\operatorname{ker} T_{G}$ is nearly $S^{*}$-invariant, and because $\operatorname{ker} A_{g}^{\theta}=$ $P_{1}\left(\operatorname{ker} T_{G}\right)$ we can use Corollary 3.2 to deduce the kernel of a truncated Toeplitz operator is nearly $S^{*}$-invariant with a defect given by $\operatorname{span}\left\{\frac{w_{1}}{z}\right\} \cap H^{2}$. With this information we can use the following result given as Theorem 3.2 in [6] (or equivalently Theorem 3.4 with $n=1$ ) to study $\operatorname{ker} A_{g}^{\theta}$.

Theorem 4.2 Let $M \subseteq H^{2}$ be a closed subspace that is nearly $S^{*}$-invariant with a finite defect $m$. Then:

1. In the case where there are functions in $M$ that do not vanish at 0 ,

$$
M=\left\{f: f(z)=f_{0}(z) k_{0}(z)+z \sum_{j=1}^{m} k_{j}(z) e_{j}(z):\left(k_{0}, \ldots, k_{m}\right) \in K\right\}
$$

where $f_{0}$ is the normalised reproducing kernel for $M$ at $0,\left\{e_{1}, \ldots e_{m}\right\}$ is any orthonormal basis for $D$, and $K$ is a closed $S^{*}$-invariant subspace of $H^{2}\left(\mathbb{D}, \mathbb{C}^{(m+1)}\right)$. Furthermore $\|f\|^{2}=\sum_{j=0}^{m}\left\|k_{j}\right\|^{2}$.

2. In the case where all functions in $M$ vanish at 0 ,

$$
M=\left\{f: f(z)=z \sum_{j=1}^{m} k_{j}(z) e_{j}(z):\left(k_{1}, \ldots, k_{m}\right) \in K\right\}
$$


with the same notation as in 1 , except that $K$ is now a closed $S^{*}$-invariant subspace of $H^{2}\left(\mathbb{D}, \mathbb{C}^{m}\right)$, and $\|f\|^{2}=\sum_{j=1}^{m}\left\|k_{j}\right\|^{2}$.

Conversely if a closed subspace $M \subseteq H^{2}$ has a representation as in 1 or 2 , the it is a nearly $S^{*}$-invariant subspace with defect $m$.

To use Theorem 4.2 we have to assume that our defect space is orthogonal to $\operatorname{ker} A_{g}^{\theta}$; we consider two separate cases. We first assume that all functions in $\operatorname{ker} A_{g}^{\theta}$ vanish at 0 . We set $O:=\operatorname{ker} A_{g}^{\theta}+\operatorname{span}\left\{\frac{w_{1}}{z}\right\}, E:=O \ominus \operatorname{ker} A_{g}^{\theta}$, we let $e$ be $P_{E}\left(\frac{w_{1}}{z}\right)$ and then $e$ is orthogonal to $\operatorname{ker} A_{g}^{\theta}$. In this construction $e \neq 0$ as this would imply $\frac{w_{1}}{z} \in \operatorname{ker} A_{g}^{\theta}=w_{1} K_{z \Phi}$ which is clearly a contradiction. Theorem 4.2 now yields

$$
\operatorname{ker} A_{g}^{\theta}=e z K_{\Psi}
$$

where multiplication by $e z$ is an isometry from $K_{\Psi}$ to $\operatorname{ker} A_{g}^{\theta}$. This expression for $\operatorname{ker} A_{g}^{\theta}$ is more familiar than $w_{1} K_{z \Phi}$ as in this case the multiplication is an isometry as opposed to a contraction. We can also relate this expression to nearly $S^{*}$-invariant subspaces. If we let $n$ be the greatest natural number such that $\frac{e}{z^{n}} \in H^{2}$ then $\frac{\operatorname{ker} A_{g}^{\theta}}{z^{n+1}}=$ $\frac{e}{z^{n}} K_{z \Psi}$, now $\frac{e}{z^{n}}(0) \neq 0$ so $\frac{\operatorname{ker} A_{g}^{\theta}}{z^{n+1}}=\frac{e}{z^{n}} K_{z \Psi}$ is a nearly $S^{*}$-invariant subspace. We can conclude the following theorem in this case.

Theorem 4.3 If $n$ is the greatest natural number such that $\operatorname{ker} A_{g}^{\theta} \subseteq z^{n} H^{2}$, then $\frac{\operatorname{ker} A_{g}^{\theta}}{z^{n}}$ is a nearly $S^{*}$-invariant subspace.

We now turn our attention to the case when not all functions in $\operatorname{ker} A_{g}^{\theta}$ vanish at 0 . In this case it must also follow that $w_{1}(0) \neq 0$ as otherwise $w_{1} K_{z \Phi}(0)=0$, so using Corollary 3.2 we must have the defect space for $\operatorname{ker} A_{g}^{\theta}=0$ so can conclude the following theorem.

Theorem 4.4 If $\operatorname{ker} A_{g}^{\theta}$ contains functions which do not vanish at 0 then it is nearly $S^{*}$-invariant.

When $\operatorname{ker} A_{g}^{\theta}$ is nearly $S^{*}$-invariant we may proceed by using Proposition 3 of the paper of Hitt [15] to show $\operatorname{ker} A_{g}^{\theta}=u K_{z \psi}$ where $u \in \operatorname{ker} A_{g}^{\theta} \ominus\left(\operatorname{ker} A_{g}^{\theta} \cap z H^{2}\right)$ is an isometric multiplier. As was noted in [13] we can call $\psi$ the associated inner function to $u$, and it is easily checked (similar to the approach in Proposition 4.1) this is an inner function such that $g u \psi=\overline{z p_{1}}+\theta p_{2}$ where $p_{1}$ is outer.

In fact using (7) we can view these two theorems as specialisations of the following theorem.

Theorem 4.5 If $f \in H^{2}$, and I is an inner function such that $f K_{I}$ is a closed subspace of $H^{2}$, then if $f(0) \neq 0$ then $f K_{I}$ is a nearly invariant subspace. If $f(0)=0$ then $f K_{I}$ is both a nearly invariant subspace multiplied by a power of $z$ and a nearly invariant subspace with a 1-dimensional defect space $\frac{f}{z}\left(K_{I} \ominus\left(K_{I} \cap z H^{2}\right)\right)$. 
Proof The only non-trivial statement to prove is if $f(0)=0$ then $f K_{I}$ is a nearly invariant subspace with a defect space $\frac{f}{z}\left(K_{I} \ominus\left(K_{I} \cap z H^{2}\right)\right)$, but this follows from

$$
\frac{f K_{I}}{z} \in \frac{f}{z}\left(K_{I} \ominus\left(K_{I} \cap z H^{2}\right)\right)+f\left(\frac{K_{I} \cap z H^{2}}{z}\right) \subseteq \frac{f}{z}\left(K_{I} \ominus\left(K_{I} \cap z H^{2}\right)\right)+f K_{I} .
$$

So under the assumptions $f \in H^{2}$ and $I$ is an inner function such that $f K_{I}$ is a closed subspace of $H^{2}$, if $f(0)=0$ then Theorem 4.5 gives us two possible approaches to decomposing $f K_{I}$.

1. Divide $f K_{I}$ by $z^{n}$ where $n \in \mathbb{N}$ is chosen such that $\frac{f}{z^{n}}(0) \neq 0$, then use the Hitt decomposition given in [15]. Then we could write $f K_{I}$ as $z^{n} u$ multiplied by some model space, where $u \in \frac{f K_{I}}{z^{n}} \ominus\left(\frac{f K_{I}}{z^{n}} \cap z H^{2}\right)$.

2. Use Theorem 3.2 in [6] with $\frac{f}{z}\left(K_{I} \ominus\left(K_{I} \cap z H^{2}\right)\right)$ as the defect space. Then we could write $f K_{I}$ as ze multiplied by some model space, where $e$ is chosen to be an element of $\frac{f}{z}\left(K_{I} \ominus\left(K_{I} \cap z H^{2}\right)\right)+f K_{I}$ orthogonal to $f K_{I}$.

In both of these cases we obtain a model space multiplied by an isometric multiplier.

Due to the similarities in the way these two decompositions are developed one might expect that the two possible ways of decomposing $f K_{I}$ might actually yield the same result. We show this is not the case and in general we have two different expressions with an example.

Example 4.1 Let $g=\frac{1}{1-\frac{z}{3}}\left(\bar{z}^{3}+z^{3}\right)$ and let $\theta=z^{4}$, we first find ker $A_{g}^{\theta}$ using linear algebra techniques. With respect to the basis $1, z, z^{2}, z^{3}, A_{g}^{\theta}$ has the matrix representation

$$
\left(\begin{array}{cccc}
\frac{1}{3^{3}} & \frac{1}{3^{2}} & \frac{1}{3} & 1 \\
\frac{1}{3^{4}} & \frac{1}{3^{3}} & \frac{1}{3^{2}} & \frac{1}{3} \\
\frac{1}{3^{5}} & \frac{1}{3^{4}} & \frac{1}{3^{3}} & \frac{1}{3^{2}} \\
1+\frac{1}{3^{6}} & \frac{1}{3^{5}} & \frac{1}{3^{4}} & \frac{1}{3^{3}}
\end{array}\right)
$$

which has reduced row echelon form given by

$$
\left(\begin{array}{llll}
1 & 0 & 0 & 0 \\
0 & 1 & 3 & 9 \\
0 & 0 & 0 & 0 \\
0 & 0 & 0 & 0
\end{array}\right)
$$

The kernel of this matrix has a basis given by

$$
\left(\begin{array}{c}
0 \\
1 \\
-\frac{1}{3} \\
0
\end{array}\right),\left(\begin{array}{c}
0 \\
0 \\
1 \\
-\frac{1}{3}
\end{array}\right) \text {, }
$$


and thus we can write $\operatorname{ker} A_{g}^{\theta}=z\left(1-\frac{z}{3}\right) K_{z^{2}}$. We now will give two different decompositions of this kernel using Theorem 4.5. Let $f=z\left(1-\frac{z}{3}\right)$ and $K_{I}=K_{z^{2}}$, then $f K_{I}=z \operatorname{span}\left\{\left(1-\frac{z}{3}\right), z\left(1-\frac{z}{3}\right)\right\}$. We first use approach 1 . It can be checked that

$$
1-\frac{z}{3} K_{z^{2}} \ominus 1-\frac{z}{3} K_{z^{2}} \cap z H^{2}
$$

has a normalised basis element given by

$$
u=\frac{3 \sqrt{910}}{91}\left(1-\frac{1}{30} z-\frac{1}{10} z^{2}\right)
$$

and so $f K_{I}$ can be written as $z u$ multiplied by some model space, which we will denote $K_{I_{1}}$. In order to find $I_{1}$ we must solve

$$
z\left(1-\frac{z}{3}\right) K_{z^{2}}=z u K_{I_{1}}
$$

but $\frac{\left(1-\frac{z}{3}\right)}{u}$ is a scalar multiple of $\frac{1}{1+\frac{3 z}{10}}$, so $K_{I_{1}}$ must be given by $\operatorname{span}\left\{\frac{1}{1+\frac{3 z}{10}} \frac{z}{1+\frac{3 z}{10}}\right\}$, therefore $I_{1}=z \frac{z+\frac{3}{10}}{1+\frac{3 z}{10}}$. So we conclude

$$
z\left(1-\frac{z}{3}\right) K_{z^{2}}=z \frac{3 \sqrt{910}}{91}\left(1-\frac{1}{30} z-\frac{1}{10} z^{2}\right) K_{z\left(\frac{z+\frac{3}{10}}{1+\frac{3 z}{10}}\right)}
$$

where multiplication by $z \frac{3 \sqrt{910}}{91}\left(1-\frac{1}{30} z-\frac{1}{10} z^{2}\right)$ is an isometry on the model space. This can be simplified to

$$
z\left(1-\frac{z}{3}\right) K_{z^{2}}=z\left(30-z-3 z^{2}\right) K_{z\left(\frac{z+\frac{1}{3}}{1+\frac{z}{3}}\right)}
$$

however in this case we no longer have the multiplication on the model space acting as an isometry. Now we use approach 2 . We must find a normalised element $e \in$ $z\left(1-\frac{z}{3}\right) K_{z^{2}}+\operatorname{span}\left\{\left(1-\frac{z}{3}\right)\right\}$, which is orthogonal to $z\left(1-\frac{z}{3}\right) K_{z^{2}}$. This can be checked to be

$$
\sqrt{\frac{729}{74620}}\left(\frac{91}{9}-\frac{1}{27} z-\frac{1}{9} z^{2}-\frac{1}{3} z^{3}\right)
$$

which means $f K_{I}$ can also be written as ze multiplied by some model space, which we will denote $K_{I_{2}}$. Now to find $I_{2}$ we must solve

$$
z\left(1-\frac{z}{3}\right) K_{z^{2}}=z e K_{I_{2}}
$$


$e$ is a scalar multiple of

$$
\left(273-z-3 z^{2}-9 z^{3}\right)=3\left(1-\frac{z}{3}\right)\left(9 z^{2}+30 z+91\right)
$$

and so $K_{I_{2}}$ must be $\operatorname{span}\left\{\frac{1}{9 z^{2}+30 z+91}, \frac{z}{9 z^{2}+30 z+91}\right\}$. We now aim to find the inner function $I_{2}$. We denote $A=\frac{1}{9 z^{2}+30 z+91}$ and $B=\frac{z}{9 z^{2}+30 z+91} . A(0)=\frac{1}{91}$, so

$$
S^{*}(A)(z)=\frac{A(z)-\frac{1}{91}}{z}=\frac{-9 z-30}{91\left(9 z^{2}+30 z+91\right)}=-\frac{30}{91} A-\frac{9}{91} B .
$$

It is clear that $S^{*}(B)=A$. We now aim to find two eigenvectors of the backwards shift operator (these are necessarily Cauchy kernels) which are in $\operatorname{span}\{A, B\}$. If we use $A, B$ as a basis for $\operatorname{span}\{A, B\}$ then the matrix representation of the backwards shift operator is given by

$$
\left(\begin{array}{cc}
-\frac{30}{91} & 1 \\
-\frac{9}{91} & 0
\end{array}\right)
$$

This has eigenvalues given by $\frac{-15 \pm 3 i \sqrt{66}}{91}$, we denote $\lambda_{1}=\frac{-15+3 i \sqrt{66}}{91}$ and $\lambda_{2}=$ $\frac{-15-3 i \sqrt{66}}{91}$, then the corresponding eigenvectors are given by $k_{\overline{\lambda_{1}}}=\frac{1}{1-\lambda_{1} z}$ and $k_{\overline{\lambda_{2}}}=$ $\frac{1}{1-\lambda_{2} z}$, so as mentioned in the introduction $I_{2}=\left(\frac{z-\overline{\lambda_{1}}}{1-\lambda_{1} z}\right)\left(\frac{z-\overline{\lambda_{2}}}{1-\lambda_{2} z}\right)$. We can conclude

$$
z\left(1-\frac{z}{3}\right) K_{z^{2}}=z \sqrt{\frac{729}{74620}}\left(\frac{91}{9}-\frac{1}{27} z-\frac{1}{9} z^{2}-\frac{1}{3} z^{3}\right) K_{\left(\frac{z-\overline{\lambda_{1}}}{1-\lambda_{1} z}\right)\left(\frac{z-\overline{\lambda_{2}}}{1-\lambda_{2} z}\right)}
$$

where multiplication by $z \sqrt{\frac{729}{74620}}\left(\frac{91}{9}-\frac{1}{27} z-\frac{1}{9} z^{2}-\frac{1}{3} z^{3}\right)$ is an isometry on the model space. Again we can simplify this to

$$
z\left(1-\frac{z}{3}\right) K_{z^{2}}=z\left(273-z-3 z^{2}-9 z^{3}\right) K\left(\frac{z-\overline{\lambda_{1}}}{1-\lambda_{1} z}\right)\left(\frac{z-\overline{\lambda_{2}}}{1-\lambda_{2} z}\right)
$$

but in this expression we no longer have the multiplication on the model space acting as an isometry. Thus approach 1 and approach 2 give different decompositions.

\section{Application to Truncated Toeplitz Operators on Multiband Spaces}

Truncated Toeplitz operators on multiband spaces are soon to be introduced in a publication which is currently in preparation by M.C. Câmara, R. O'Loughlin, and J.R. Partington. They are defined (on the unit circle) as follows. Let $g \in L^{\infty}$, let $\phi, \psi$ be unimodular functions in $L^{\infty}$ such that $\phi K_{\theta} \perp \psi K_{\theta}$, we define the multiband space 
$M:=\phi K_{\theta} \oplus \psi K_{\theta}$. The truncated Toeplitz operator on $M$ denoted $A_{g}^{M}: M \rightarrow M$ is defined by

$$
A_{g}^{M}(f)=P_{M}(g f),
$$

where $P_{M}$ is the orthogonal projection on to $M$. These operators have applications in speech processing and as a special case, if we let $\phi=\bar{\theta}$ and $\psi=\theta$ we recover a (disc variation) of the Paley-Wiener space.

We write $K_{\theta}\left(\mathbb{D}, \mathbb{C}^{n}\right) \subseteq H^{2}\left(\mathbb{D}, \mathbb{C}^{n}\right)$ to mean the vectors of length $n$ with each coordinate taking entries in $K_{\theta}$. To study truncated Toeplitz operators on multiband spaces we first consider the truncated Toeplitz operator $A_{G}^{\theta}$ acting on $K_{\theta}\left(\mathbb{D}, \mathbb{C}^{2}\right)$, where

$$
G=\left(\begin{array}{ll}
g_{11} & g_{12} \\
g_{21} & g_{22}
\end{array}\right)
$$

has each entry in $L^{\infty}$. Using the unitary map $U: M \rightarrow K_{\theta}\left(\mathbb{D}, \mathbb{C}^{2}\right)$ where

$$
U\left(\phi f_{1}+\psi f_{2}\right)=\left(\begin{array}{l}
f_{1} \\
f_{2}
\end{array}\right)
$$

one can show that any truncated Toeplitz operator on a multiband space is unitarily equivalent to $A_{G}^{\theta}$ for a certain choice of $G$. Thus we turn our attention to studying $\operatorname{ker} A_{G}^{\theta}$.

If we define

$$
\mathcal{G}=\left(\begin{array}{cccc}
\bar{\theta} & 0 & 0 & 0 \\
0 & \bar{\theta} & 0 & 0 \\
g_{11} & g_{12} & \theta & 0 \\
g_{21} & g_{22} & 0 & \theta
\end{array}\right),
$$

then it is easily checked that

$$
\left(\begin{array}{l}
p \\
q \\
r \\
s
\end{array}\right) \in \operatorname{ker} T_{\mathcal{G}}
$$

if and only if $p, q \in K_{\theta}$ and

$$
\left(\begin{array}{ll}
g_{11} & g_{12} \\
g_{21} & g_{22}
\end{array}\right)\left(\begin{array}{l}
p \\
q
\end{array}\right)+\theta\left(\begin{array}{l}
r \\
s
\end{array}\right) \in \overline{H_{0}^{2}} \oplus \overline{H_{0}^{2}}
$$


So $\left(\begin{array}{l}p \\ q\end{array}\right) \in \operatorname{ker} A_{G}^{\theta}$, and likewise given $\left(\begin{array}{l}p \\ q\end{array}\right) \in \operatorname{ker} A_{G}^{\theta}$ there exists $\left(\begin{array}{l}r \\ s\end{array}\right) \in H^{2}$ with $\left(\begin{array}{l}p \\ q \\ r \\ s\end{array}\right) \in \operatorname{ker} T_{\mathcal{G}}$. Keeping the same notation as Theorem 3.1 we let $W=$ $\operatorname{ker} T_{\mathcal{G}} \ominus\left(\operatorname{ker} T_{\mathcal{G}} \cap z H^{2}\left(\mathbb{D}, \mathbb{C}^{4}\right)\right)$, and let $W_{1} \ldots W_{r}$ be an orthonormal basis for $W$, as previously mentioned $r \leq 4$. Toeplitz kernels are nearly $S^{*}$-invariant so by Corollary 3.2 we know $P_{2}\left(\operatorname{ker} T_{\mathcal{G}}\right)=\operatorname{ker} A_{G}^{\theta}$ is nearly $S^{*}$-invariant with defect space $\left(\frac{\operatorname{span}\left\{P_{2}\left(W_{1}\right), \ldots P_{2}\left(W_{r}\right)\right\}}{z} \cap H^{2}\left(\mathbb{D}, \mathbb{C}^{2}\right)\right)$. We now try to find the dimension of this defect space. For $F$ a set of functions we denote

$$
F(0)=\{f(0): f \in F\} .
$$

Lemma 5.1 $\operatorname{dim} \operatorname{ker} T_{\mathcal{G}}(0)=\operatorname{dim} W=\operatorname{dim} W(0)$.

Proof Lemma 3.9 in [17] shows that $\operatorname{dim} \operatorname{ker} T_{\mathcal{G}}(0)=\operatorname{dim} W$, Lemma 3.3 shows that $W_{1}(0) \ldots W_{r}(0)$ are linearly independent and clearly $W_{1}(0) \ldots W_{r}(0)$ span $W(0)$.

We first consider the case when $\operatorname{dim} W=4$, in this case by Lemma 5.1 we have $W(0)=\mathbb{C}^{4}$. We have a correspondence between the matrix $\left[W_{1}, W_{2}, W_{3}, W_{4}\right]$ and a 4-by-4 matrix taking values in $\mathbb{C}$ given by

$$
\left[W_{1}, W_{2}, W_{3}, W_{4}\right] \mapsto\left[W_{1}(0), W_{2}(0), W_{3}(0), W_{4}(0)\right]
$$

We also know by Lemma 5.1 that $W_{1}(0), W_{2}(0), W_{3}(0), W_{4}(0)$ are a basis for $\mathbb{C}^{4}$, so there exists a sequence of column operations we can perform to $W_{1}(0), W_{2}(0), W_{3}(0)$, $W_{4}(0)$ which yields the identity matrix. If we perform the same column operations to $\left[W_{1}, W_{2}, W_{3}, W_{4}\right]$ we will obtain a matrix

$$
\left[\tilde{W}_{1}, \tilde{W}_{2}, \tilde{W}_{3}, \tilde{W}_{4}\right]
$$

which has the property that $\left[\tilde{W}_{1}(0), \tilde{W}_{2}(0), \tilde{W}_{3}(0), \tilde{W}_{4}(0)\right]$ is equal to the identity matrix. The linear independence of $\tilde{W}_{1}(0), \tilde{W}_{2}(0), \tilde{W}_{3}(0), \tilde{W}_{4}(0)$ implies linear independence of $\tilde{W}_{1}, \tilde{W}_{2}, \tilde{W}_{3}, \tilde{W}_{4}$, and so $\tilde{W}_{1}, \tilde{W}_{2}, \tilde{W}_{3}, \tilde{W}_{4}$ span $W$. It is now clear that $\left(\frac{\operatorname{span}\left\{P_{2}\left(W_{1}\right), \ldots P_{2}\left(W_{4}\right)\right\}}{z} \cap H^{2}\left(\mathbb{D}, \mathbb{C}^{2}\right)\right)$ is given by $\frac{\operatorname{span}\left\{P_{2}\left(\tilde{W}_{3}\right) P_{2}\left(\tilde{W}_{4}\right)\right\}}{z}$, and so when $\operatorname{dim} W=4$, we have $\operatorname{ker} A_{g}^{\theta}$ is nearly invariant with the 2-dimensional defect space $\frac{\operatorname{span}\left\{P_{2}\left(\tilde{W}_{3}\right) P_{2}\left(\tilde{W}_{4}\right)\right\}}{z}$.

We now consider the case when $\operatorname{dim} W=3$, in this case $W(0)$ is a 3-dimensional subspace of $\mathbb{C}^{4}$. We again have a correspondence

$$
\left[W_{1}, W_{2}, W_{3}\right] \mapsto\left[W_{1}(0), W_{2}(0), W_{3}(0)\right]
$$


In this case we can perform column operations to $W_{1}(0), W_{2}(0), W_{3}(0)$ to obtain a matrix which takes one of the following four forms (here we denote $x_{1}, x_{2}, x_{3}$ to be some unknown unspecified values in $\mathbb{C}$ ),

$$
\left(\begin{array}{ccc}
1 & 0 & 0 \\
0 & 1 & 0 \\
0 & 0 & 1 \\
x_{1} & x_{2} & x_{3}
\end{array}\right),\left(\begin{array}{ccc}
1 & 0 & 0 \\
0 & 1 & 0 \\
x_{1} & x_{2} & x_{3} \\
0 & 0 & 1
\end{array}\right),\left(\begin{array}{ccc}
1 & 0 & 0 \\
x_{1} & x_{2} & x_{3} \\
0 & 1 & 0 \\
0 & 0 & 1
\end{array}\right),\left(\begin{array}{ccc}
x_{1} & x_{2} & x_{3} \\
1 & 0 & 0 \\
0 & 1 & 0 \\
0 & 0 & 1
\end{array}\right) .
$$

As in the previous case if we perform these same column operations which yield one of the above to the matrix $\left[W_{1}, W_{2}, W_{3}\right]$ we will obtain a matrix

$$
\left[\tilde{W}_{1}, \tilde{W}_{2}, \tilde{W}_{3}\right] .
$$

By the same arguments made previously we can deduce $\tilde{W}_{1}, \tilde{W}_{2}, \tilde{W}_{3}$ span $W$. This means $\left(\frac{\operatorname{span}\left\{P_{2}\left(W_{1}\right), \ldots P_{2}\left(W_{3}\right)\right\}}{z} \cap H^{2}\right)$ is contained in $\frac{\operatorname{span}\left\{P_{2}\left(\tilde{W}_{2}\right) P_{2}\left(\tilde{W}_{3}\right)\right\}}{z} \cap H^{2}\left(\mathbb{D}, \mathbb{C}^{2}\right)$, and so when $\operatorname{dim} W=3$, we have $\operatorname{ker} A_{G}^{\theta}$ is nearly invariant with (at most) 2dimensional defect space

$$
\frac{\operatorname{span}\left\{P_{2}\left(\tilde{W}_{2}\right) P_{2}\left(\tilde{W}_{3}\right)\right\}}{z} \cap H^{2}\left(\mathbb{D}, \mathbb{C}^{2}\right) .
$$

In the case when $\operatorname{dim} W \leq 2$ it is clear from Corollary 3.2 that the defect space of $\operatorname{ker} A_{G}^{\theta}$ has dimension at most 2. So we can conclude the following theorem.

Theorem $5.2 \operatorname{ker} A_{G}^{\theta}$ is a nearly $S^{*}$-invariant subspace with defect 2.

We now give an example to show that in general 2 is the smallest dimension of defect space, i.e. it is not true that for all inner functions $\theta$ and matrix symbols $G$ that $\operatorname{ker} A_{G}^{\theta}$ has a 1-dimensional defect.

Example 5.1 Following the unitary equivalence we mentioned earlier we consider an operator of the form

$$
A_{G}^{\theta}=\left(\begin{array}{cc}
A_{g}^{\theta} & A_{g \bar{\phi} \psi}^{\theta} \\
A_{g \bar{\psi} \phi}^{\theta} & A_{g}^{\theta}
\end{array}\right),
$$

where $g \in L^{\infty}, \theta$ is an inner function and $\phi, \psi \in L^{\infty}$ are unimodular functions such that $\phi K_{\theta} \perp \psi K_{\theta}$. These conditions ensure that $A_{G}^{\theta}$ is indeed unitarily equivalent to a truncated Toeplitz operator a multiband space. Let $\theta=z^{2}, \phi=z, \psi=z^{4}$, $g=2 \bar{z}^{2}+z+2 z^{4}$. We identify the basis of $K_{\theta}\left(\mathbb{D}, \mathbb{C}^{2}\right)$ with a basis of $\mathbb{C}^{4}$ in the following way $\left(\begin{array}{l}1 \\ 0\end{array}\right) \mapsto\left(\begin{array}{l}1 \\ 0 \\ 0 \\ 0\end{array}\right),\left(\begin{array}{l}z \\ 0\end{array}\right) \mapsto\left(\begin{array}{l}0 \\ 1 \\ 0 \\ 0\end{array}\right),\left(\begin{array}{l}0 \\ 1\end{array}\right) \mapsto\left(\begin{array}{l}0 \\ 0 \\ 1 \\ 0\end{array}\right),\left(\begin{array}{l}0 \\ z\end{array}\right) \mapsto\left(\begin{array}{l}0 \\ 0 \\ 0 \\ 1\end{array}\right)$, then $A_{G}^{\theta}$ 
has the following matrix representation

$$
\left(\begin{array}{llll}
0 & 0 & 0 & 0 \\
1 & 0 & 2 & 0 \\
0 & 0 & 0 & 0 \\
2 & 0 & 1 & 0
\end{array}\right) .
$$

Thus ker $A_{G}^{\theta}$ is given by the span of $\left(\begin{array}{l}z \\ 0\end{array}\right)$ and $\left(\begin{array}{l}0 \\ z\end{array}\right)$, which is clearly nearly $S^{*}$-invariant with defect 2.

For a multiband space $M:=\phi K_{\theta} \oplus \psi K_{\theta}$ using our unitary equivalence by $U$ we can write

$$
\operatorname{ker} A_{g}^{M}=U^{*} \operatorname{ker}\left(\begin{array}{cc}
A_{g}^{\theta} & A_{g \bar{\phi} \psi}^{\theta} \\
A_{g \bar{\psi} \phi}^{\theta} & A_{g}^{\theta}
\end{array}\right)
$$

Combining this with Theorem 5.2 and Theorem 3.4 gives a decomposition for $\operatorname{ker} A_{g}^{M}$ in terms of $S^{*}$-invariant subspaces.

\section{Application to Dual Truncated Toeplitz Operators}

It is easily checked that in $L^{2}$ we have $K_{\theta}^{\perp}=\overline{H_{0}^{2}} \oplus \theta H^{2}$. We denote $Q$ to be the orthogonal projection $Q: L^{2} \rightarrow\left(K_{\theta}\right)^{\perp}$. Throughout this section we assume $g \in L^{\infty}$. The dual truncated Toeplitz operator $D_{g}^{\theta}:\left(K_{\theta}\right)^{\perp} \rightarrow\left(K_{\theta}\right)^{\perp}$ is defined by

$$
f \mapsto Q(g f) .
$$

Theorem 6.6 in [8] shows that for a symbol $g$ that is invertible in $L^{\infty}$ we have $\operatorname{ker} D_{g}^{\theta}=$ $g^{-1} \operatorname{ker} A_{g^{-1}}^{\theta}$, so given our observation (7) under the condition that $g$ is invertible in $L^{\infty}$ we can write $\operatorname{ker} D_{g}^{\theta}$ as an $L^{2}$ function multiplied by a model space. We now aim to use similar recursive methods that were used to prove Theorem 3.4 to obtain a decomposition theorem for $\operatorname{ker} D_{g}^{\theta}$.

Throughout this section we assume that $\operatorname{ker} D_{g}^{\theta}$ is finite dimensional.

We define $A:=\left\{f \in \operatorname{ker} D_{g}^{\theta}: g f \in K_{\theta} \cap z H^{2}\right\}$ and $C:=\operatorname{ker} D_{g}^{\theta} \cap \overline{\left(H_{0}^{2}\right.} \oplus$ $\left.\theta z H^{2}\right) \cap A$, then using orthogonal decomposition we can write

$$
\operatorname{ker} D_{g}^{\theta}=C \oplus\left(\operatorname{ker} D_{g}^{\theta} \ominus C\right) \text {. }
$$

Lemma 6.1 If ker $D_{g}^{\theta} \subseteq C$ then $\operatorname{ker} D_{g}^{\theta}=\{0\}$. 
Proof Suppose we have a non-zero $f \in \operatorname{ker} D_{g}^{\theta} \subseteq C$, then by construction of $C$ we must have $\frac{f}{z} \in \operatorname{ker} D_{g}^{\theta} \subseteq C$. Iterating this we can obtain $\frac{f}{z^{n}} \in \operatorname{ker} D_{g}^{\theta}$ for all $n \in \mathbb{N}$, which can't be true as given $n$ sufficiently large $\frac{g f}{z^{n}} \notin H^{2}$.

Corollary 6.2 For any $\operatorname{ker} D_{g}^{\theta} \neq\{0\}$ we have $1 \leq \operatorname{dim}\left(\operatorname{ker} D_{g}^{\theta} \ominus C\right) \leq 2$.

Proof If ker $D_{g}^{\theta} \neq\{0\}$ then Lemma 6.1 shows that $1 \leq \operatorname{dim}\left(\operatorname{ker} D_{g}^{\theta} \ominus C\right)$. Let $F_{1}$ be the orthogonal projection of $\bar{g} k_{0}$ on to $\operatorname{ker} D_{g}^{\theta}$ and $F_{2}$ be the orthogonal projection of $\theta k_{0}$ on to ker $D_{g}^{\theta}$, where $k_{0}$ is the reproducing kernel at 0 , then $\operatorname{ker} D_{g}^{\theta} \ominus C$ is generated by $F_{1}, F_{2}$. Indeed if $f \in \operatorname{ker} D_{g}^{\theta}$ and $f$ is orthogonal to $F_{1}, F_{2}$ then

$$
\left\langle f, F_{1}\right\rangle=\left\langle g f, k_{0}\right\rangle=0,
$$

so $f \in A$, and

$$
\left\langle f, F_{2}\right\rangle=\left\langle\bar{\theta} f, k_{0}\right\rangle=0,
$$

so we also have $P(\bar{\theta} f) \subseteq z H^{2}$, so $f \in C$.

Consider $g$ ker $D_{g}^{\theta}=g C \oplus\left(g \operatorname{ker} D_{g}^{\theta} \ominus g C\right)$, by Corollary 6.2 we must have $g \operatorname{ker} D_{g}^{\theta} \ominus g C$ is at most 2-dimensional. If $g \operatorname{ker} D_{g}^{\theta} \ominus g C$ is 2-dimensional then we denote its orthonormal basis elements by $g f_{0}, g h_{0}$. Then for all $f \in \operatorname{ker} D_{g}^{\theta}$ using orthogonal projections and the observation that $\frac{C}{z} \subseteq \operatorname{ker} D_{g}^{\theta}$ we can write

$$
g f=\lambda_{0} g f_{0}+\mu_{0} g h_{0}+z g f_{1},
$$

where $g f_{1} \in g \operatorname{ker} D_{g}^{\theta}$, and furthermore

$$
\|g f\|^{2}=\left|\lambda_{0}\right|^{2}+\left|\mu_{0}\right|^{2}+\left\|g f_{1}\right\|^{2} .
$$

In a similar process to Theorem 3.4 we can iterate this process starting with $g f_{1}$ to obtain

$$
g f=\sum_{i=0}^{N} g f_{0} \lambda_{i} z^{i}+\sum_{j=0}^{N} g h_{0} \mu_{j} z^{j}+z^{N+1} g f_{N+1},
$$

with

$$
\|g f\|^{2}=\sum_{i=0}^{N}\left|\lambda_{i}\right|^{2}+\sum_{j=0}^{N}\left|\mu_{j}\right|^{2}+\left\|g f_{N+1}\right\| .
$$

Following the argument laid out in Sect. 3 to deduce (3) we can deduce that in the $H^{2}$ norm $\left\|g f_{N+1}\right\| \rightarrow 0$ as $N \rightarrow \infty$, then $\left\|g f_{N+1}\right\|$ must also converge to 0 in the $L^{1}$ 
norm, and so in the $L^{1}$ norm we must have

$$
g f=\lim _{N \rightarrow \infty}\left(\sum_{i=0}^{N} g f_{0} \lambda_{i} z^{i}+\sum_{j=0}^{N} g h_{0} \mu_{j} z^{j}\right) .
$$

Now two applications of Hölder's inequality shows the $L^{1}$ limit of $\sum_{i=0}^{N} g f_{0} \lambda_{i} z^{i}+$ $\sum_{j=0}^{N} g h_{0} \mu_{j} z^{j}$ is equal to $g f_{0} \sum_{i=0}^{\infty} \lambda_{i} z^{i}+g h_{0} \sum_{j=0}^{\infty} \mu_{j} z^{j}$, where $\sum_{i=0}^{\infty} \lambda_{i} z^{i}$, $\sum_{j=0}^{\infty} \mu_{j} z^{j}$ are limits in the $H^{2}$ sense. So we may write

$$
g f=g f_{0} \sum_{i=0}^{\infty} \lambda_{i} z^{i}+g h_{0} \sum_{j=0}^{\infty} \mu_{j} z^{j}
$$

and furthermore by taking limits in (8) we can deduce

$$
\|g f\|_{H^{2}}^{2}=\sum_{i=0}^{\infty}\left|\lambda_{i}\right|^{2}+\sum_{i=0}^{\infty}\left|\mu_{i}\right|^{2} .
$$

Mimicking the argument from Sect. 3 between (5) and (6) we can say $f \in \operatorname{ker} D_{g}^{\theta}$ if and only if

$$
g f=\left(\begin{array}{lll}
g f_{0} & g h_{0}
\end{array}\right)\left(\begin{array}{l}
k_{0} \\
k_{1}
\end{array}\right)
$$

where $\left(\begin{array}{l}k_{0} \\ k_{1}\end{array}\right)$ lies in a closed $S^{*}$-invariant subspace of $H^{2}\left(\mathbb{D}, \mathbb{C}^{2}\right)$. With obvious modifications for when $\operatorname{dim} \operatorname{ker} D_{g}^{\theta} \ominus C=1$ we can deduce the following theorem.

Theorem 6.3 1. If $\operatorname{dim}\left(g \operatorname{ker} D_{g}^{\theta} \ominus g C\right)=2$ then

$$
g \operatorname{ker} D_{g}^{\theta}=\left(g f_{0} g h_{0}\right) K
$$

where $K$ is a closed $S^{*}$-invariant subspace of $H^{2}\left(\mathbb{D}, \mathbb{C}^{2}\right), g f_{0}, g h_{0}$ are orthonormal basis elements of $\left(g \operatorname{ker} D_{g}^{\theta} \ominus g C\right)$ and for $f \in \operatorname{ker} D_{g}^{\theta}$ we have $\|g f\|_{H^{2}}^{2}=$ $\left\|k_{0}\right\|_{H^{2}}^{2}+\left\|k_{1}\right\|_{H^{2}}^{2}$.

2. If $\operatorname{dim}\left(g \operatorname{ker} D_{g}^{\theta} \ominus g C\right)=1$ then

$$
g \operatorname{ker} D_{g}^{\theta}=g f_{0} K_{\chi z}
$$

where $\chi$ is some inner function, $g f_{0}$ is a normalised element of ( $g \operatorname{ker} D_{g}^{\theta} \ominus g C$ ) and for $f \in \operatorname{ker} D_{g}^{\theta}$ we have $\|g f\|_{H^{2}}^{2}=\|k\|_{H^{2}}^{2}$.

Cancelling the $g$ and using the same notation as the previous theorem we obtain the following. 
Corollary 6.4 1. If $\operatorname{dim}\left(\operatorname{ker} D_{g}^{\theta} \ominus C\right)=2$ then

$$
\operatorname{ker} D_{g}^{\theta}=\left(\begin{array}{ll}
f_{0} & h_{0}
\end{array}\right)\left(\begin{array}{l}
k_{0} \\
k_{1}
\end{array}\right)
$$

2. If $\operatorname{dim}\left(\operatorname{ker} D_{g}^{\theta} \ominus C\right)=1$ then

$$
\operatorname{ker} D_{g}^{\theta}=f_{0} K_{\chi z}
$$

Acknowledgements The author is grateful to the EPSRC for financial support. The author is grateful to Professor Partington for his valuable comments.

\section{Compliance with ethical standards}

Conflicts of interest The authors declare that they have no conflict of interest.

Code availability Data sharing not applicable to this article as no datasets were generated or analysed during the current study.

Open Access This article is licensed under a Creative Commons Attribution 4.0 International License, which permits use, sharing, adaptation, distribution and reproduction in any medium or format, as long as you give appropriate credit to the original author(s) and the source, provide a link to the Creative Commons licence, and indicate if changes were made. The images or other third party material in this article are included in the article's Creative Commons licence, unless indicated otherwise in a credit line to the material. If material is not included in the article's Creative Commons licence and your intended use is not permitted by statutory regulation or exceeds the permitted use, you will need to obtain permission directly from the copyright holder. To view a copy of this licence, visit http://creativecommons.org/licenses/by/4.0/.

\section{References}

1. Agler, J., McCarthy, J.E.: Pick Interpolation and Hilbert Function Spaces, vol. 44. American Mathematical Society, New York (2002)

2. Baranov, A., Bessonov, R., Kapustin, V.: Symbols of truncated Toeplitz operators. J. Funct. Anal. 261(12), 3437-3456 (2011)

3. Baranov, A., Chalendar, I., Fricain, E., Mashreghi, J., Timotin, D.: Bounded symbols and reproducing kernel thesis for truncated Toeplitz operators. J. Funct. Anal. 259(10), 2673-2701 (2010)

4. Câmara, M.C., Partington, J.R.: Spectral properties of truncated Toeplitz operators by equivalence after extension. J. Math. Anal. Appl. 433(2), 762-784 (2016)

5. Chalendar, I., Chevrot, N., Partington, J.R.: Nearly invariant subspaces for backwards shifts on vectorvalued Hardy spaces. J. Oper. Theory 63(2), 403-415 (2010)

6. Chalendar, I., Gallardo-Gutiérrez, E.A., Partington, J.R.: A Beurling theorem for almost-invariant subspaces of the shift operator. J. Oper. Theory 83(2), 321-331 (2020)

7. Cima, J.A., Ross, W.T.: The Backward Shift on the Hardy Space. American Mathematical Society, New York (2000)

8. Câmara, M.C., Kliś-Garlicka, K., Łanucha, B., Ptak, M.: Invertibility, Fredholmness and kernels of dual truncated Toeplitz operators. arXiv:1912.13266 (2019)

9. Câmara, M.C., Ross, W.T.: The dual of the compressed shift. arXiv:2001.02587 (2020)

10. Câmara, M.C., Partington, J.R.: Scalar-type kernels for block Toeplitz operators. J. Math. Anal. Appl. 489(1), 124111 (2020)

11. Ding, X., Sang, Y.: Dual truncated Toeplitz operators. J. Math. Anal. Appl. 461(1), 929-946 (2018)

12. Duren, P.L.: Theory of $H^{p}$ spaces. Pure Appl. Math 38, 74 (1970) 
13. Hartmann, A., Seip, K.: Extremal functions as divisors for kernels of Toeplitz operators. J. Funct. Anal. 202(2), 342-362 (2003)

14. Hayashi, E.: The kernel of a Toeplitz operator. Integral Equ. Oper. Theory 9(4), 588-591 (1986)

15. Hitt, D.: Invariant subspaces of $H^{2}$ of an annulus. Pacific J. Math. 134(1), 101-120 (1988)

16. Nikolski, N.K.: Operators, Functions, and Systems-An Easy Reading: Hardy, Hankel, and Toeplitz, vol. 1. American Mathematical Society, New York (2002)

17. O’Loughlin, R.: Toeplitz kernels and the backward shift. J. Math. Anal. Appl. 492(2), (2020)

18. Peller, V.V.: Hankel Operators and Their Applications. Springer Monographs in Mathematics. Springer, Berlin (2003)

19. Sarason, D.: Nearly invariant subspaces of the backward shift. In Contributions to Operator Theory and its Applications, pp. 481-493. Springer (1988)

20. Sarason, D.: Algebraic properties of truncated Toeplitz operators. Oper. Matrices 1(4), 491-526 (2007)

Publisher's Note Springer Nature remains neutral with regard to jurisdictional claims in published maps and institutional affiliations. 